\title{
The integration of single fiber reflectance (SFR) spectroscopy during endoscopic ultrasound-guided fine needle aspirations (EUS-FNA) in pancreatic masses: a feasibility study
}

\author{
Paulien L. Stegehuis ${ }^{\mathrm{a}}$, Leonora S. F. Boogerd ${ }^{\mathrm{a}}$, Akin Inderson ${ }^{\mathrm{a}}$, Roeland A. Veenendaal ${ }^{\mathrm{a}}$, Bert A. \\ Bonsing $^{\mathrm{a}}$, Arjen Amelink ${ }^{\mathrm{b}}$, Alexander L. Vahrmeijer ${ }^{\mathrm{a}}$, Jouke Dijkstra ${ }^{\mathrm{a}}$, Dominic J. Robinson ${ }^{\mathrm{c}}$ \\ ${ }^{a}$ Leiden University Medical Center, Albinusdreef 2, Leiden, the Netherlands, ${ }^{\mathrm{b}}$ TNO, Stieltjesweg 1, \\ Delft, the Netherlands, ' Erasmus Medical Center, 's-Gravendijkwal 230, Rotterdam, the Netherlands
}

\begin{abstract}
EUS-FNA can be used for pathological confirmation of a suspicious pancreatic mass. However, performance depends on an on-site cytologist and time between punction and final pathology results can be long. SFR spectroscopy is capable of extracting biologically relevant parameters (e.g. oxygenation and blood volume) in real-time from a very small tissue volume at difficult locations. In this study we determined feasibility of the integration of SFR spectroscopy during EUSFNA procedures in pancreatic masses.
\end{abstract}

Patients with benign and malignant pancreatic masses who were scheduled for an EUS-FNA were included. The working guide wire inside the 19 gauge endoscopic biopsy needle was removed and the sterile single fiber (300 $\mu \mathrm{m}$ core and 700 $\mu \mathrm{m}$ outer diameter, wide-angle beam, NA 0.22) inserted through the needle. Spectroscopy measurements in the visiblenear infrared wavelength region $(400-900 \mathrm{~nm}$ ) and autofluorescence measurements (excitation at $405 \mathrm{~nm}$ ) were taken three times, and subsequently cytology was obtained. Wavelength dependent optical properties were compared to cytology results.

We took measurements in 13 patients with corresponding cytology results (including mucinous tumor, ductal adenocarcinoma, neuroendocrine tumor, and pancreatitis). In this paper we show the first analyzed results comparing normal pancreatic tissue with cancerous tissue in the same patient. We found a large difference in blood volume fraction, and blood oxygenation was higher in normal tissue.

Integration of SFR spectroscopy is feasible in EUS-FNA procedures, the workflow hardly requires changes and it takes little time. The first results differentiating normal from tumor tissue are promising.

Keywords: Single fiber reflectance spectroscopy, pancreatic tumor, endoscopy

\section{INTRODUCTION}

Pancreatic cancer is the fourth leading cause of cancer deaths, leading to 330,000 deaths worldwide yearly, and the incidence is still increasing in developed coutries. ${ }^{1}{ }^{2}$ The five year survival rate is less than $5 \%{ }^{3}$ A big contributor to the low five year survival rate is the late detection of pancreatic cancer: early-stage disease is usually clinically silent, and when the presentation of symptoms occur (such as abdominal pain, weight loss and jaundice), the tumor is already locally too involved or metastasized. ${ }^{4}$

For the diagnosis and staging of pancreatic cancer, a contrast enhanced computed tomography (CT) scan is the imaging modality of choice. ${ }^{5}$ Endoscopic ultrasound fine needle aspiration (EUS-FNA) is also used to diagnose pancreatic lesions; it combines ultrasound imaging with cytological findings. It is useful when nothing is seen on CT, but pancreatic cancer is suspected, and it is also required when tissue diagnosis is needed before the start of radiation or chemotherapy. ${ }^{3}$ The sensitivity and accuracy for malignancy range from $75-92 \%$ and from $79-92 \%$, respectively. ${ }^{5}$ However, the

Endoscopic Microscopy XI; and Optical Techniques in Pulmonary Medicine III, edited by Guillermo J. Tearney, Thomas D. Wang, Melissa J. Suter, Stephen Lam, Matthew Brenner, Proc. of SPIE Vol. 9691 96910J · @ 2016 SPIE · CCC code: 1605-7422/16/\$18 · doi: 10.1117/12.2212623 
sensitivity drops to $54 \%$ in patients with chronic pancreatitis ${ }^{6}$, and the negative predictive value was estimated at $60-70 \%$ in a systematic review ${ }^{7}$.

A novel alternative method to assess pancreatic tumors is EUS-FNA with an incorporated fiber optical reflectance device, Single Fiber Reflectance (SFR) spectroscopy., ${ }^{8,9}$ SFR spectroscopy is a spectroscopic technique, capable of extracting biologically relevant parameters from a very small tissue volume. Reflectance spectra obtained by SFR spectroscopy contain information about tissue absorption and scattering properties. This fiberoptic technique, is able to determine the local microvascular saturation, the amount of blood present, the average vascular diameter and a parameter related to the scattering properties of tissue in real-time. These parameters are believed to be relevant for cancer diagnosis. To facilitate measurements of these parameters during endoscopic ultrasound guided fine needle aspiration procedures, we will use a fiberoptic probe that fits through the needle of a standard EUS-FNA device.

The aim of this feasibility study is to evaluate the SFR spectroscopy incorporated into the EUS-FNA procedure to differentiate benign from malignant pancreatic tumors. The second aim is to measure the wavelength dependent optical properties to distinguish between malignant and benign pancreatic tissue.

\section{MATERIALS \& METHODS}

\subsection{Subjects}

This study was approved by the Medical Ethics Committee of the Leiden University Medical Center (Leiden, the Netherlands). Patients who were scheduled for an EUS-FNA for a suspected pancreatic mass were eligible to participate in this study. Informed consent was obtained from all patients prior to the procedure.

\subsection{Measurement setup}

Individual SF reflectance and fluorescence spectra were measured sequentially, at the same tissue location using a custom-made instrument illustrated schematically in Figure 1. The SF probe consisted of a single optical fiber for both light delivery and light collection, with a core diameter $(300 \mu \mathrm{m})$ small enough to be fed through the 19 gauge endoscopic biopsy needle channel. The proximal end of the single fiber probe connects to a quadfurcated fiber, with one arm leading to a tungsten-halogen lamp (HL-2000-FHSA; Ocean Optics; Duiven, NL); one arm leading to a spectrophotometer (SD 2000; Ocean Optics; Duiven, NL); one arm to a $405 \mathrm{~nm}$ laser light source and the final arm to a second spectrograph (QE-65000, Ocean Optics; Duiven, NL) that is filtered using a $450 \mathrm{~nm}$ long pass filter to block 405 $\mathrm{nm}$ excitation light. The distal end of the single fiber probe, which is in contact with the tissue during measurement, is polished at an angle of $15^{\circ}$ to minimize the collection of specular reflection that is caused by refractive index mismatch between the fiber and tissue. During reflectance measurements, light in the VIS-NIR wavelength range (400-900 nm) travels from the lamp to the single fiber probe tip, exits the fiber and scatters throughout the tissue. Light that is remitted from the tissue and is collected by the fiber returns a wavelength-dependent reflectance intensity $\left(R_{\mathrm{SF}}\right)$. During fluorescence measurements tissue autofluorescence was collected under $405 \mathrm{~nm}$ excitation. A calibration procedure, described in detail previously in a paper by Hoy et al. ${ }^{10}$, was used to account for internal reflections and for variability in lamp- and laser-specific output and fiber-specific transmission characteristics.

\subsection{Examination procedure}

The EUS-FNA procedure was performed as standard, and the gastroenterologist decided whether a 19 gauge endoscopic biopsy needle would be used. If so, the guide wire of that needle was removed before introduction into the endoscope, and a sterilized single-use fiber was introduced into the 19 gauge needle. Before FNA, at least 3 reflectance and fluorescence measurements were acquired.

\subsection{Mathematical analysis of spectra}

SF reflectance spectra $\left(R_{S F}\right)$ were analyzed using a mathematical model, published previously ${ }^{11}$, that describes the wavelength-dependent effects of scattering and absorption on the reflectance intensity collected by the SF device in tissue. The model is given by 


$$
R_{S F}\left(d_{f}\right)=\left\lfloor a_{1}\left(\frac{\lambda}{\lambda_{0}}\right)^{-a_{2}}\right\rfloor\left(d_{f}\right) \mathrm{e}^{-\mu_{a, T_{\text {Tis }}}\langle L\rangle}
$$

where the term in square brackets is a background scattering model that follows a Mie theory wavelength dependence; here fitted parameters describing the Mie amplitude $\left(a_{1}\right)$ and Mie slope $\left(a_{2}\right)$. The model describes attenuation due to absorption via a modified Beer-Lambert law which includes the product of the absorption coefficient within the tissue, $\mu_{\mathrm{a}, \text { Tiss }}$ and $<\mathrm{L}>$ the effective photon path length, given by

$$
\frac{\langle L\rangle}{d_{f}}=\frac{1.54}{\left(\mu_{s}(\lambda) d_{f}\right)^{0.18}\left(0.64+\mu_{a} d_{f}\right)^{0.64}}
$$

The absorption coefficient of tissue is expressed as the sum of the absorption of blood $\mu_{\mathrm{a}, \text { blood }}$ and the absorption of bilirubin $\mu_{a}^{b i j}$ :

$$
\mu_{\mathrm{a}, \pi \mathrm{s}}(\lambda)=\mu_{\mathrm{a}, \text { brood }}(\lambda)+\mu_{\mathrm{a}}^{\mathrm{bil}}(\lambda)
$$

The absorption coefficient of blood can be described as the product of the specific blood absorption coefficients and blood volume fraction:

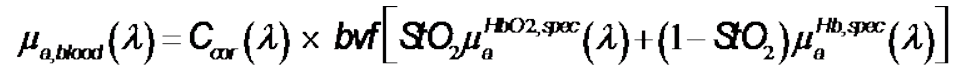

where $\mathrm{StO}_{2}$ is the blood oxygen saturation, bvf is blood volume fraction assuming the concentration of hemoglobin in whole blood to be $150 \mathrm{~g} / \mathrm{L}$, and $\mu_{a}^{\boldsymbol{H O O 2 , p e c}}$ and $\mu_{a}^{\boldsymbol{H}, \mathbf{s p e c}}$ are the specific absorption coefficients of oxygenized and deoxygenized blood, respectively. $\mathrm{C}_{\text {cor }}$ is the correction factor that accounts for flattening of spectral features resulting from the inhomogeneous distribution of blood in tissue and the confinement of blood in vessels. For whole blood, the correction factor is given by:

$$
C_{c o r}=\left\{1-\exp \left[\mu_{a, b l o o d}(\lambda) D_{v}\right]\right\} /\left[\mu_{a, \text { blood }}(\lambda) D_{v}\right]
$$

which is related to the average vessel diameter $D_{v}$. The total tissue absorption $\mu_{a, \text { tiss }}$ can then be described as the absorption of blood $\mu_{\mathrm{a} \text {,blood, }}$, dependend on blood volume fraction, and the absorption of bilirubin, which is the product of the specific absorption coefficient $\mu_{a}^{b i l, p s c}$ and the bilirubin concentration $\mathrm{C}_{\mathrm{bil}}$ :

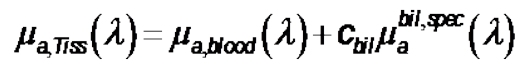

Fitting the measured data to equation 1 yields estimate for the micro-vascular saturation, (StO2), blood volume fraction $(\rho)$ the average vessel diameter $\left(D_{v}\right)$ the bilibrubin concentration, the Mie slope and the scattering amplitude.

In an analogous manner fluorescence spectra were analyzed to recover an estimate of the fluorescence intensity corrected for the measured average absorption coefficient at the excitation and emission wavelength using a modified form of the Beer-Lambert law;

$$
F_{S F}=F_{S F}^{0} e^{\left(-\mu_{a, a g g}\left(L_{S F L}\right)\right.}
$$

Here, $\mu_{\mathrm{a}, \text { avg }}$ is the average $\mu_{\mathrm{a}}$ at the excitation and emission wavelength and the fluorescence photon path length $\mathrm{L}_{\mathrm{SFFL}}$ is defined as

$$
\left\langle L_{\text {SFR }}\right\rangle=\langle Z\rangle \frac{1+\kappa \sqrt{\left(\mu_{\text {sang }} d_{f}\right)}}{1+\left(\mu_{\text {a, } w g} d_{f}\right)}
$$

where $\mu_{a, a v g}$ is the average $\mu_{s, \text { avg }}$ at excitation and emission wavelength, $d_{\mathrm{f}}$ is the fiber diameter and $\kappa$ represents a single fitted parameter which has a value of $\kappa=1.81 \pm 0.01 . \mathrm{Z}$ is the effective sampling depth defined as; 


$$
\langle Z\rangle=d_{f} A_{2}\left(\mu_{\text {s,ang }} d_{f}\right)^{-A_{3}}
$$

In this equation, $A_{2}$ and $A_{3}$ are determined empirically to be $0.71 \pm 0.01$ and $0.36 \pm 0.01$ respectively. We have previously developed a model to relate $\mathrm{F}_{\mathrm{SF}}{ }^{0}$ to the intrinsic fluorescence spectrum $\mu_{\mathrm{a}}{ }^{\mathrm{f}} \mathrm{Q}^{\mathrm{f}}$

$$
F_{R=i o}^{\mathscr{F}}=\frac{F_{S \mathscr{F}}^{0}}{T X P L} \approx\left(\frac{\lambda_{x}}{\lambda_{m}}\right) \mu_{a}^{f} Q^{f}\left\langle\phi_{x}^{v}\right\rangle\left\langle H_{m}^{v}\right\rangle\langle V\rangle
$$

In this equation, $\langle\mathrm{V}\rangle,\left\langle\mu_{\mathrm{x}}{ }^{\mathrm{V}}\right\rangle$ and $\left\langle\mathrm{H}_{\mathrm{m}}{ }^{\mathrm{V}}\right\rangle$ are the effective sampling volume, excitation fluence and escape probability respectively. It is important to note that this approach, previously described in detail ${ }^{12}$ relies on an accurate determination of $\mu_{s, a v g}$. Since this is unknown in the present implementation of SFF spectroscopy and we have assumed that that the Mie scattering parameters $a_{1}$ and $a_{2}$ are equal to 1 at $800 \mathrm{~nm}$. In this way the autofluorescence we report correctly accounts for differences in $\mu_{\mathrm{a}}$ but does not account for differences in $\mu_{\mathrm{s}}$.

\section{RESULTS}

Figures 1 and 2 display representative SFR spectra collected from tissue of measurements in the same patient. On endoscopic examination an irregular hypo-echogenic, heterogeneous, low-vascular mass was seen in the pancreatic head with a size of $25 \mathrm{~mm}$. Acquired cytology indicated a malignancy. In follow-up this was confirmed by histology of a hepatic lesion: a adenocarcinoma of pancreatic biliary origin.

The measurement corresponding to Figure 1 was taken at a location in close proximity to the suspected mass. This was indicated by the gastroenterologist and supported by ultrasound images, but no cytology was taken at this location. Cytology was acquired at the spectrum corresponding to Figure 2, which indicated a malignancy.

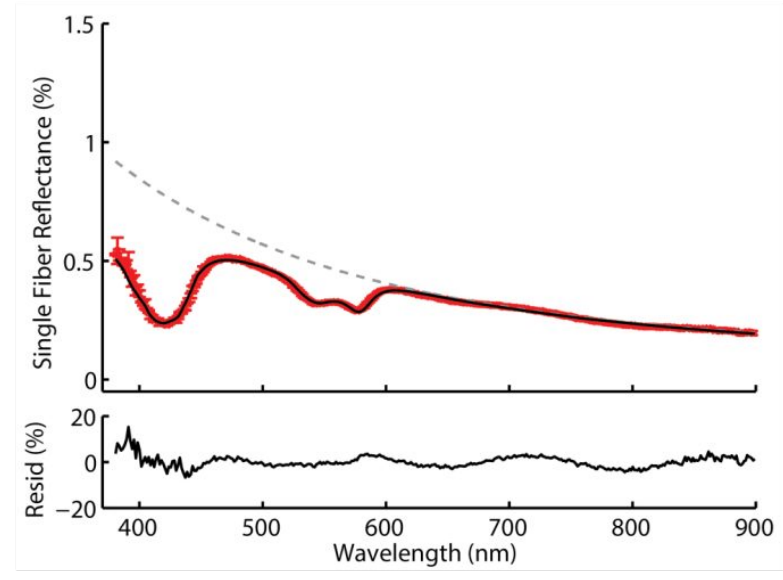

Figure 1. SFR spectrum of normal pancreatic tissue, the actual (red) and fitted (black) spectrum. The residual showed underneath represents the difference between measured and fitted spectrum. 


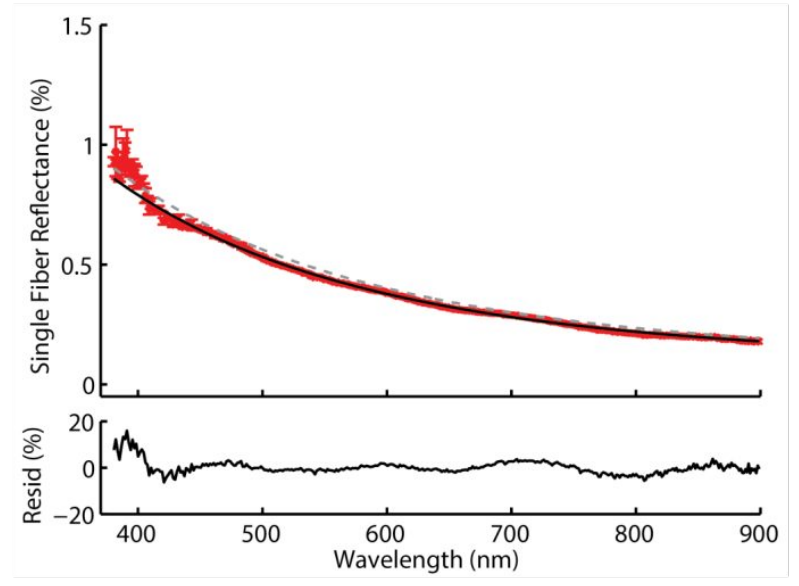

Figure 2. SFR spectrum of pancreatic tumor tissue, the actual (red) and fitted (black) spectrum. The residual showed underneath represents the difference between measured and fitted spectrum.

Table 1. Mean physiological and morphological parameters extracted from SFR measurements. (Data is displayed as mean \pm SD).

\begin{tabular}{lll}
\hline Parameter & Normal & Malignant \\
\hline Blood volume fraction $(\%)$ & $4.5 \pm 0.22$ & $0.087 \pm 0.07$ \\
Hemoglobin saturation $(\%)$ & $64.9 \pm 3.9$ & $45.8 \pm 20.5$ \\
Average vessel diameter $(\mu \mathrm{m})$ & $2.9 \pm 0.5$ & $0.01 \pm 6$ \\
\hline
\end{tabular}

Table 1 displays the extracted biologically relevant parameters for measurements taken at both locations. As the blood volume fraction at the malignant side is so low, the average vessel diameter is not meaningful here.

\section{DISCUSSION AND CONCLUSION}

These preliminary results of in-vivo pancreatic measurements show that not only taking SFR spectroscopy measurements during an EUS-FNA procedure is feasible, but also that based on the biological parameters extracted, a distinction can be made between normal and malignant tissue.

SFR Spectroscopy measurements only added approximately 5-10 minutes to the standard procedure, as the fiber could be pre-loaded into the needle. With more experience and omitting fluorescence measurements, this time could be shortened.

We found a higher blood volume fraction and higher hemoglobin saturation at the normal site. These results are in line with studies previously performed. Using the same technique, Kanick et al. ${ }^{13}$, found a significantly higher hemoglobin saturation and blood volume fraction in normal compared to metastatic mediastinal lymph nodes. To our knowledge no spectroscopic measurements have been performed in-vivo on pancreatic tissue, but pancreatic cancer is characterized by abundant fibrous stroma and hypovascularity ${ }^{14}$, which is consistent with our findings.

In conclusion, this study showed the feasibility of the incorporation of SFR spectroscopy measurements during EUSFNA. This could be of use clinically to guide the gastroenterologist to the location of interest, where a biopsy can then be taken. The first results look promising, but only consist of few measurements, so no firm conclusions can yet be drawn. 


\section{ACKNOWLEDGEMENTS}

This research has received funding from the European Union Seventh Framework Program FP7-ICT-2011-8 under grant agreement number 318729 (CAReIOCA project).

\section{REFERENCES}

[1] Torre, L.A., Bray, F., Siegel, R.L., Ferlay, J., Lortet-tieulent, J., and Jemal, A., “Global Cancer Statistics, 2012,” CA: a cancer journal of clinicians. 65(2), 87-108 (2015).

[2] Wada, K., Takoari, K., and Traverso, W.L., “Screening for pancreatic cancer,” Surgical Clinics of North America 95(5), 1041-1052 (2015).

[3] Hidalgo, M., "Pancreatic cancer.," The New England journal of medicine 362(17), 1605-17 (2010).

[4] Vincent, A., Herman, J., Schulick, R., Hruban, R.H., and Goggins, M., "Pancreatic cancer,” The Lancet 378(9791), 607-620 (2011).

[5] Iglesias-garcía, J., Lindkvist, B., Lariño-noia, J., and Domínguez-muñoz, J.E., "The role of endoscopic ultrasound ( EUS ) in relation to other imaging modalities in the differential diagnosis between mass forming chronic pancreatitis , autoimmune pancreatitis and ductal pancreatic adenocarcinoma,” Rev Esp Enferm Dig 104, 315-321 (2012).

[6] Fritscher-Ravens, A., Brand, L., Knöfel, W.T., Bobrowski, C., Topalidis, T., Thonke, F., de Werth, A., and Soehendra, N., "Comparison of endoscopic ultrasound-guided fine needle aspiration for focal pancreatic lesions in patients with normal parenchyma and chronic pancreatitis.," The American journal of gastroenterology 97(11), 2768-75 (2002).

[7] Hartwig, W., Schneider, L., Diener, M.K., Bergmann, F., Büchler, M.W., and Werner, J., "Preoperative tissue diagnosis for tumours of the pancreas.,” The British journal of surgery 96(1), 5-20 (2009).

[8] Kanick, S.C., Sterenborg, H.J.C.M., and Amelink, a, "Empirical model of the photon path length for a single fiber reflectance spectroscopy device.," Optics express 17(2), 860-71 (2009).

[9] Kanick, S.C., Robinson, D.J., Sterenborg, H.J.C.M., and Amelink, a, "Monte Carlo analysis of single fiber reflectance spectroscopy: photon path length and sampling depth.," Physics in medicine and biology 54(22), 6991-7008 (2009).

[10] Hoy, C.L., Gamm, U. a., Sterenborg, H.J.C.M., Robinson, D.J., and Amelink, a., "Use of a coherent fiber bundle for multi-diameter single fiber reflectance spectroscopy,” in Biomed. Opt. Express 3(10), p. 2452 (2012).

[11] van Leeuwen-van Zaane, F., Gamm, U. a, van Driel, P.B. a a, Snoeks, T.J. a, de Bruijn, H.S., van der Ploeg-van den Heuvel, a, Mol, I.M., Löwik, C.W.G.M., Sterenborg, H.J.C.M., et al., "In vivo quantification of the scattering properties of tissue using multi-diameter single fiber reflectance spectroscopy.,” Biomedical optics express 4(5), 696-708 (2013).

[12] Kanick, S.C., Robinson, D.J., Sterenborg, H.J.C.M., and Amelink, A., "Extraction of intrinsic fluorescence from single fiber fluorescence measurements on a turbid medium.," Optics letters 37(5), 948-50 (2012).

[13] Kanick, S.C., van der Leest, C., Djamin, R.S., Janssens, A., Hoogsteden, H., Sterenborg, H.J.C.M., Amelink, A., and Aerts, J., "Characterization of Mediastinal Lymph Node Physiology In Vivo by Optical Spectroscopy during Endoscopic Ultrasound-Guided Fine Needle Aspiration,” J Thorac Oncol 5(7), 981-987 (2010).

[14] Miura, F., Takada, T., Amano, H., Yoshida, M., Furui, S., and Takeshita, K., "Diagnosis of pancreatic cancer.," HPB : the official journal of the International Hepato Pancreato Biliary Association 8, 337-342 (2006). 